\title{
Estimation of Surface Potential of Lymphocytes from Patients with Leukemia Using Kelvin Probe Mode
}

\author{
E. A. Sladkova and M. Yu. Skorkina \\ Belgorod State National Research University, Belgorod, 308015 Russia \\ e-mail: evgenija-sladkova00@rambler.ru \\ Received January 9, 2013; in final form, November 25, 2013
}

\begin{abstract}
The feasibilities of the Kelvin probe mode were shown to estimate a surface potential of lymphocytes from the patients with leukemia. Having compared the values of the surface potential obtained by the traditional method of microelectrophoresis and by the Kelvin probe mode, it was established that the use of the atomic force microscope made it possible to obtain data which were of specified diagnostic importance while studying lymphocyte functional status at different stages in leucopoiesis and indicated progression of the pathological process.
\end{abstract}

Keywords: surface potential, lymphocytes, leukemia, atomic force microscopy

DOI: $10.1134 / \mathrm{S} 0006350914020250$

\section{INTRODUCTION}

Bioelectric processes exert substantial influence on the activity of physiological systems [1]. On the cellular level they are realized at the expense of work of ion channels of the membrane, participating in the formation of surface potential. The electric system of the cell plays the key role in the functioning of its receptors [2], maintenance of a state of equilibrium between integrative physiological processes: proliferation, differentiation and programmed cell death [3]. In a series of experimental works it is shown that a change of membrane surface potential is characteristic of activation of lymphocytes and may be immediately connected with processes of their blast-transformation [4].

For studying bioelectric processes in single cells, use is made of a direct method of measuring the surface potential with the aid of a microelectrode technique, which is connected with certain methodical difficulties and great time expenditure [5]. A whole series of advantages is possessed by optical indicators - probes reacting to changes of membrane potential by different fluorescence intensity of shifting of the spectral maximum [5,6]. The disadvantages of such probes come to be their nonspecific binding with membranes structures of the cell, fading, autofluorescence [7]. One of the promising methods that may replace the microelectrode technique and fluorescent probes is presented by the Kelvin probe mode used in atomic force microscopy (AFM) technologies [8].

The aim of the present work was to evaluate the surface potential of lymphocytes from leukemia patients by the Kelvin probe mode.

\section{EXPERIMENTAL}

Investigations were executed on the basis of the Chair of anatomy and physiology of living organisms, Belgorod National Research University. The object of investigation was served by peripheral blood lymphocytes of patients with acute lymphoblastic leukemia, chronic lymphoblastic leukemia, acute myeloblastic leukemia and acute lymphoblastic leukemia in remission, 15 persons in each group. In the quality of control we used lymphocytes of 50 healthy donors.

Preparation of samples of blood cells for investigation was conducted by the following scheme: blood was centrifuged $10 \mathrm{~min}$ at $1500 \mathrm{rpm}$, collecting the lower part of plasma rich in leukocytes and the leukocyte ring. Leukocytes were twice washed with isotonic buffer solution (Dulbecco solution pH 7.4). A leukocyte suspension for measuring the surface potential was prepared by the following means (patent No. 2027188) [9]. Blood white cells were washed with isotonic solution of sodium chloride for $5 \mathrm{~min}$, then fixation was conducted with $0.25 \%$ solution of glutaric aldehyde for $20 \mathrm{~min}$. When the indicated time elapsed, the suspension of leukocytes was twice washed with isotonic solution of sodium chloride for $5 \mathrm{~min}$ each and preparations were prepared on defatted metal support.

Measurement of surface potential was actualized on an AFM "INTEGRA Vita" (configuration on the base of inverted optical microscope Olympus IX-71) in the Kelvin probe mode, using a cantilever with current-conducting titanium coating of series NSG03/TiN (Nanoworld, USA). The currently applied Kelvin probe mode is based on a two-pass pro- 
(a)
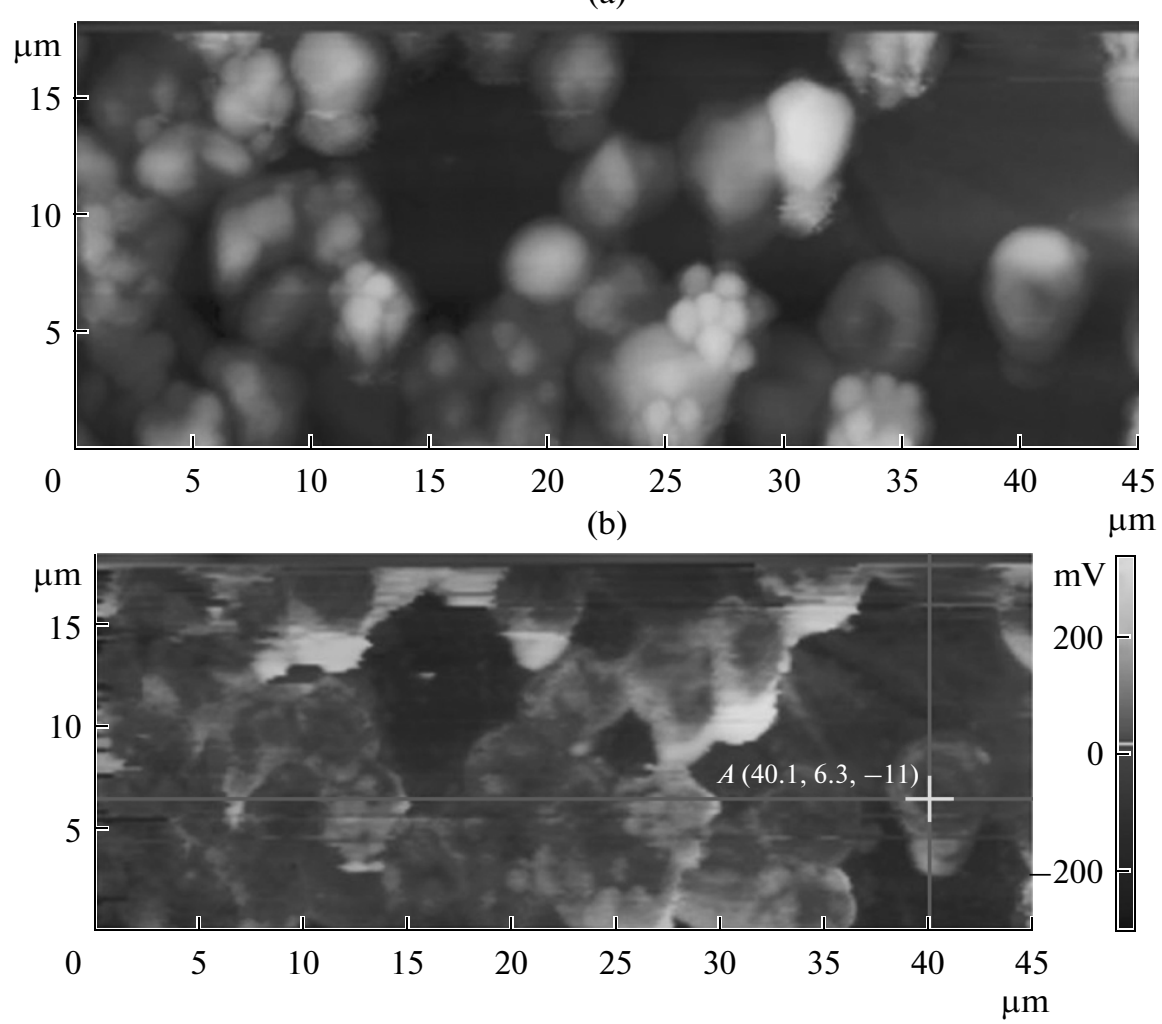

(a) Scan of lymphocytes (first pass), (b) distribution of surface potential of the surface of lymphocytes (second pass).

tocol. In the first pass, the relief of sample surface is determined with the use of half-contact mode of AFM scanning. In the second pass this relief is tracked during passing the probe over the sample at some height for determining the surface electric potential (see figure) [10] with consequent processing of the scans in the Nova program (Zelenograd, Russia).

For assessing the reliability of the new method in the conducted investigation we collated the magnitudes of surface potential of lymphocytes from healthy donors measured by the Kelvin probe mode and the commonly known method of cell microelectrophoresis in a horizontal microchamber [11]. Electrophoresis was actualized in phosphate buffer solution, $\mathrm{pH} 7.4$ at a dilution of lymphocyte suspension $1: 800$. In the basis of the electrophoretic method there lies measurement of the velocity of mobility of blood cells in an electric field with subsequent calculation of $\xi$-potential by the Helmholtz-Smoluchowski formula [12]. We have measured the $\xi$-potential of lymphocytes of 10 healthy donors ( 50 cells in every sample). Statistical treatment of the obtained results was actualized with the aid of the Student's $t$-test.

\section{RESULTS AND DISCUSSION}

In a preliminary series of tests we have measured with the aid of microelectrophoresis the magnitudes of surface potential of lymphocytes from healthy donors $(-47.43 \pm 2.31 \mathrm{mV})$. They turned out to be close to magnitudes $(-46.14 \pm 6.42 \mathrm{mV})$ measured with the Kelvin probe mode (see Table 1). In this way, the values of surface potential obtained with application of AFM can be deemed adequate.

By the Kelvin probe mode it is established that for lymphocytes of patients with various forms of leukemia, characteristic is an increase of surface potential, and consequently, of hyperpolarization of the cell membrane. For lymphocytes of acute lymphoblastic leukemia patients, characteristic was the greatest increase in surface potential, while for cells of acute myeloblastic leukemia patients - the smallest as compared with the values of surface potential of the lymphocytes of healthy donors (Table 2). The revealed peculiarity is consistent with literature data and, probably, comes as a result of growth of $\mathrm{K}^{+}$-conductivity during blast-transformation of lymphocytes [4]. The distinction of values of the surface potential off cells from patients with various forms of leukemia is connected with peculiarities of the course of disease. Probably, growth of the surface potential of lymphocytes from acute myeloblastic leukemia patients comes as a consequence of stimulatory impact of tumor factors released by transformed cells of the myeloid lineage [13, 14]. 
Table 1. Magnitudes of surface potential of lymphocytes measured by method of microelectrophoresis and in the Kelvin probe mode

\begin{tabular}{l|c|c}
\hline \multicolumn{1}{c|}{ Measured values } & Method of microelectrophoresis & Kelvin probe mode \\
\hline Surface potential, $\mathrm{mV}$ & $-47.43 \pm 2.31$ & $-46.14 \pm 6.42$ \\
Time of lymphocyte motion, $\mathrm{s}$ & 60.00 & - \\
Distance passed by lymphocytes, $\mu \mathrm{m}$ & $4.31 \pm 0.52$ & - \\
\hline
\end{tabular}

Table 2. Surface potential of peripheral blood lymphocytes

\begin{tabular}{l|l}
\hline \multicolumn{1}{c|}{ Experimental group } & Surface potential, $\mathrm{mV}$ \\
\hline Healthy donors & $-46.13 \pm 6.41$ \\
Patients with acute lymphoblastic leukemia & $-17.72 \pm 1.64 *$ \\
Patients with chronic lymphoblastic leukemia & $-27.63 \pm 1.17 *, * *$ \\
Patients with acute myeloblastic eukemia & $-34.72 \pm 2.37 *, * *, * * *$ \\
Patients with acute lymphoblastic leukemia in remissiona & $-39.91 \pm 3.10^{* * *}$ \\
\hline
\end{tabular}

* Statistically reliable differences of surface potential values compared with healthy donors by Student's test at $p<0.05$, ** statistically reliable differences of values compared with acute lymphoblastic leukemia patients by Student's test at $p<0.05$, *** statistically reliable differences of values compared with chronic lymphoblastic leukemia patients by Student's test at $p<0.05$.

In this way, the simplicity of technical execution, reduction to an minimum of factors capable of lowering the reliability of obtained results, analysis of a large mass of cell at one time and the possibility of evaluating the surface potential of a single cell allow supposing that the Kelvin probe mode may find broad application in experimental biological investigations in the quality of indicator of the degree of expression of a pathological process during investigation of the functional status of lymphocytes at different step of leukemigenesis.

\section{ACKNOWLEDGMENTS}

The work was supported by the Ministry of Education and Science of the Russian Foundation, agreement 14.132.21.1320.

\section{REFERENCES}

1. A. A. Yarygin, Patol. Fisiol. Eksperim. Terapiya 2, 38 (1998).

2. O. V. Tarnopol'skaya, Candidate's Dissertation in Physics \& Math (MGU, Moscow, 1994).
3. M. F. Nikonova, M. M. Litvina, M. I. Varfolomeeva, et al., Immunologiya 2, 20 (1999).

4. A. A. Vereninov, E. V. Gusev, O. M. Kazakova, et al., Tsitologiya 33 (11), 78 (1991).

5. N. S. Kavok, M. Yu. Malyukina, I. A. Borovoi, and N. L. Pogrebnyak, Vestn. Biofiziki 21 (2), 45 (2008).

6. L. V. Johuson, M. L. Walsh, and L. B. Chen, Proc. Natl. Acad. Sci. USA 77, 990 (1980).

7. S. Salvioli and A. Ardizroni, FEBS Lett., 77-82 (1997).

8. D. V. Allison, N. P. Mortensen, K. J. Sulivan, and M. J. Doktycz, Adv. Rev. 2, 619 (2010).

9. Yu. A. Sheremetev, G. I. Makin, and F. Yu. Suslov, RF Patent 2027188 G01N33/49. Appl. No. 4947820/14 of 26.04.1991.

10. User Manual "Probe Nanolaboratory Integra Vita" (NTMDT, Zelenograd, 2006).

11. S. G. Karasev, O. V. D’yachenko, O. V. Popova, et al., Klin. Lab. Diagnost. 11, 16 (1997).

12. G. M. Stolyar, Vopr. Eksperim. Biofiz. 6, 117 (1975).

13. O. V. Lebedinskaya, Doctoral Dissertation in Medicine (St. Petersburg, 2006).

14. O. V. Lebedinskaya, Sovrem. Naukoemkie Tekhnol. 7, 49 (2005). 